\title{
An Approach of Nonlinear Model Multi-step-ahead Predictive Control Based on SVM
}

\author{
Weimin Zhong, Daoying Pi, and Youxian Sun \\ National Laboratory of Industrial Control Technology, \\ Institute of Modern Control Engineering, Zhejiang University, Hangzhou 310027, China \\ \{wmzhong, dypi, yxsun\}@iipc.zju.edu.cn
}

\begin{abstract}
In this paper, a support vector machine (SVM) with polynomial kernel function based nonlinear model multi-step-ahead controller is presented. A SVM based multi-step-ahead predictive model is established by black-box identification. And control law is obtained by numerical and optimization methods respectively. The effect of controller is demonstrated on a recognized benchmark problem. Simulation results show that multi-step-ahead predictive controller can be well applied to nonlinear system with good performance.
\end{abstract}

\section{Introduction}

Model predictive control (MPC) is a class of control algorithms in which a process model is used to predict and optimize process performance ${ }^{[1]}$. Now, attention has turned to nonlinear model predictive control (NMPC) ${ }^{[2]}$ because many industrial processes are intrinsically nonlinear. Recently a new kind of learning machine called $\mathrm{SVM}^{[3,4]}$, which is well used for function regression and time series prediction, can be used for nonlinear system identification and system control. This paper puts forward a new approach to NMPC based on SVM with quadratic polynomial kernel function. The paper is organized as follows: In section 2, a SVM with polynomial kernel function based multi-step-ahead predictive model and control algorithm for nonlinear systems are discussed. Simulation results on a recognized benchmark problem are given in section 3. In section 4, some conclusions are put forward. For more details about SVM regression with polynomial kernel function, see references ${ }^{[3,4]}$.

\section{SVM Based NMPC}

\subsection{Nonlinear Predictive Control Model}

Assume the $j$-step-ahead ( $j=1 \cdots P, P$ is prediction horizon) nonlinear model can be described by SVM form:

$$
\begin{gathered}
y_{m}(k+j)=f\left(I_{k+j}\right)=f[y(k+j-1), y(k+j-2), \cdots, y(k-n+j), \\
u(k+j-1), u(k+j-2), \cdots, u(k-n+j)] \\
=\sum_{i=1}^{n s v} a_{i}\left(I_{i}^{\prime} \cdot I_{k+j}+1\right)^{2}+b \\
\text { s.t. } \quad u_{\min } \leq u \leq u_{\max }
\end{gathered}
$$


Where $n, m$ are determined by approximation accuracy. Support values $a_{i}(i=1, \cdots, n s v)$ and bias value $b$ can be gotten through learning according to $d$ pairs training data $\left\{I_{s}, y\right\}(s=1, \cdots, d) . I^{\prime}$ is the set of support vectors from $I_{s}$. And assume when $j>M, u(k+j-1)=u(k+M-1), M$ is control horizon.

Introduce feedback correction to deal with the model error and disturbance, so the $j$-step-ahead closed-loop predictive output at time $k$ is:

$$
y_{p}(k+j)=y_{m}(k+j)+h_{j} e(k)
$$

Where $h_{j}$ is the error correcting coefficient, $e(k)=y(k)-y_{m}(k)$.

\subsection{Nonlinear Predictive Controller}

Select optimization objective function with moving horizon as below:

$$
\min J(k)=\sum_{j=1}^{P} q_{j}\left[y_{r}(k+j)-y_{p}(k+j)\right]^{2}
$$

Where $q_{j}$ are weighting coefficients, $y_{r}(k+j)$ is reference value from trajectory:

$$
y_{r}(k+j)=a_{r}^{j} y(k)+\left(1-a_{r}^{j}\right) y_{s p} \quad y_{r}(k)=y(k)
$$

Where $a_{r}$ is the coefficient related with system's robustness and $y_{s p}$ is set point value.

\section{Case of $P=M$ :}

In this case, nonlinear optimization problem (3) contains $M$ manipulate variables and $M$ equality constraint of model outputs. Use known $y_{p}(k-1+j / k-1)$ replace unknown $y(k-1+j)$, where $y_{p}(k-1+j / k-1)$ is the closed-loop predictive output.

$$
\begin{aligned}
\min J(k)= & \underbrace{q_{1}\left[y_{r}(k+1)-y_{p}(k+1)\right]^{2}}_{J_{1}(k) \text { contains } u(k)}+\underbrace{q_{2}\left[y_{r}(k+2)-y_{p}(k+2)\right]^{2}}_{J_{2}(k) \text { contains } u(k), u(k+1)} \\
& +\cdots+\underbrace{q_{M}\left[y_{r}(k+M)-y_{p}(k+M)\right]^{2}}_{J_{M}(k) \text { contains } u(k), u(k+1), \cdots, u(k+M)}
\end{aligned}
$$

It's a common sense that the first-step controller output $u(k)$ outweighs the others, so we can first minimize $J_{1}(k)$ to get $u(k)$, and then substitute $u(k)$ into $J_{2}(k)$ to get $u(k+1)$. Recursively, we can get all $u(k+j-1)$.

$$
\frac{\partial J_{j}(k)}{\partial u(k+j-1)}=2 q_{j}\left[y_{p}(k+j)-y_{r}(k+j)\right] \frac{\partial y_{p}(k+j)}{\partial u(k+j-1)}=0
$$

It's a cubic equation with only one unknown bounded variable, and it's easily solved. 


\section{Case of $P>M$ :}

Consider nonlinear optimization problem (3) with $P>M$, this is a nonlinear optimal problem with equality constraint of model output and boundary constraint of controller output. In this paper, Nelder-Mead simplex direct search method is used to optimize and obtain $M$ unknown variables. And we will use solutions obtained by algorithm in section 2.2.1 as starting point.

\section{Experimental and Simulation Results}

The example is a benchmark problem used by Narendra \& Parthasarathy ${ }^{[5]}$, and further studied by Kambhampati ${ }^{[2]}$ using a Gaussian neural network to fulfill the onestep-ahead predictive control. Set $C=10000, \varepsilon=0.001, h_{j}=1, q_{j}=1$.

$$
y(k+1)=\frac{y(k)}{1+y^{2}(k)}+u^{3}(k)
$$

where $y$ is the plant output and $u \in[-2,2]$ is the input.

150 pairs data generated by applying by a series of random numbers between $[-2,2]$ are used to train the SVM predictive model with $m=n=5$. And set $y_{s p}=1.5$ and $a_{r}=0.9$. Fig. 2 is the system output and controller output with $P=2, M=2$. Fig. 3 is the system output and controller output with $P=4, M=2$. In both cases, a disturbance rejection $(\mathrm{d}=0.1)$ is added when system is in steady state at time $\mathrm{k}=100$, and the output response will track the set point well again quickly.

\section{Conclusions}

In this paper two cases of SVM with polynomial kernel function based nonlinear model multi-step-ahead predictive control strategies are investigated. The effect of both controllers is demonstrated by a benchmark problem. The analysis and experiment results show that the control strategy will provide a robust stable control of nonlinear systems with good performance in keeping reference trajectory and disturbance-rejection.
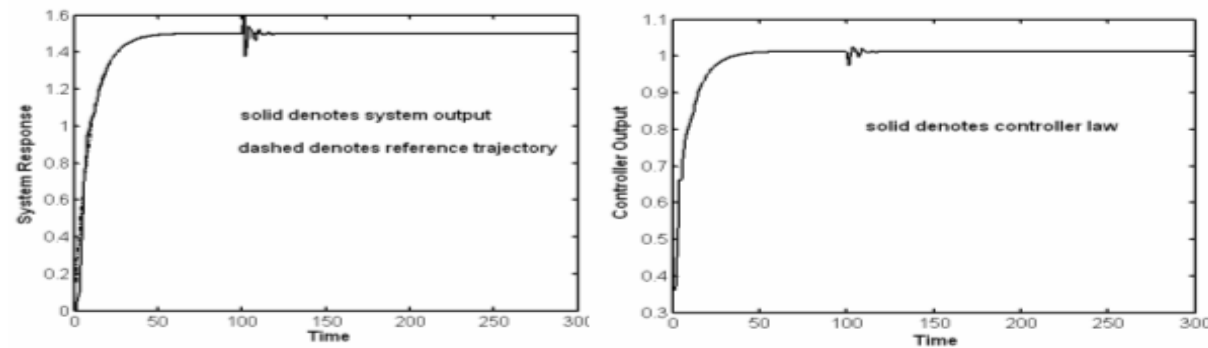

Fig. 2. System output and controller output with $P=2, M=2$ 

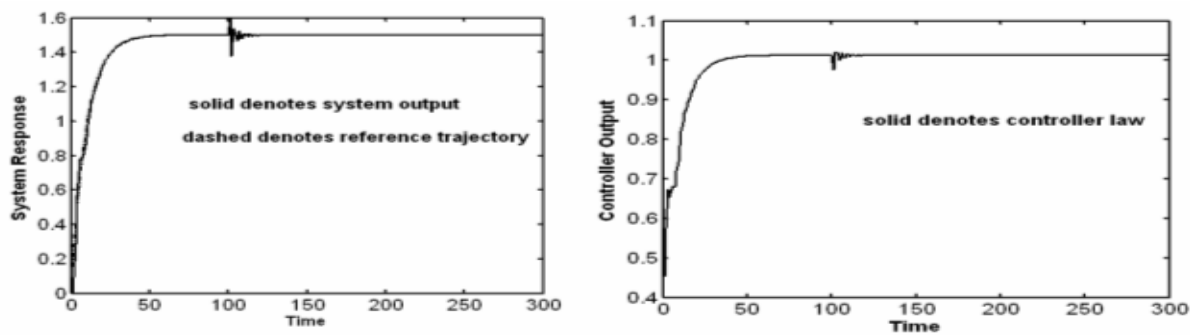

Fig. 3. System output and controller output with $P=4, M=2$

\section{Acknowledgment}

This work is supported by China 973 Program under grant No.2002CB312200.

\section{References}

1. Shu D.Q.: Predictive control system and its application. China Machine Press, Beijing, 1996

2. Kambhampati C., Mason J.D., Warwick K.: A stable one-step-ahead predictive control of non-linear systems. Automatica, Vol.36 (2000) 485-495

3. Vapnik V.N.: The nature of statistical learning theory. Springer-Verlag, New York, USA (1995)

4. Cortes C.: Prediction of generalization ability in learning machines (PhD thesis), University of Rochester, New York, USA (1995)

5. Narendra K.S., Parthasarathy K. : Identification and control of dynamic systems using neural networks. IEEE Trans. Neural Networks, vol.1, (2000) 4-27 